\title{
miR-9 inhibition of neuronal apoptosis and expression levels of apoptosis genes Bcl-2 and Bax in depression model rats through Notch pathway
}

\author{
PENG XIAO, XIAOMING ZHANG, YANFEI LI, ZHONGYI MA, SHUPING SI and XINXUE GAO
}

Department of Psychiatry, Jining Psychiatric Hospital, Jining, Shandong 272051, P.R. China

Received September 9, 2019; Accepted November 6, 2019

DOI: $10.3892 / \mathrm{etm} .2019 .8228$

\begin{abstract}
Effects of micro ribonucleic acid (miR)-9 on neuronal apoptosis and expression levels of apoptosis genes B-cell lymphoma-2 (Bcl-2) and $\mathrm{Bcl}-2$ associated $\mathrm{X}$ protein (Bax) in depression model rats, as well as its regulatory mechanism, were investigated. Thirty Sprague-Dawley rats were randomly divided into control group $(n=10)$, model group $(n=10)$ and miR-9 inhibitor group $(\mathrm{n}=10)$. The rat model of depression was established using the chronic stress method. The learning and memory abilities of rats were detected via water maze test, the neuronal morphology of the brain was detected using hematoxylin and eosin (H\&E) staining, and the levels of serum Bcl-2 and Bax were determined using the enzyme-linked immunosorbent assay (ELISA) kits. Moreover, the neuronal apoptosis in the brain was determined through terminal deoxynucleotidyl transferase-mediated dUTP nick end labeling (TUNEL) assay, and the protein levels of Notch1 and Hes1 in brain tissues were measured via western blot analysis. Compared with the control group, the rats in the model group presented significantly decreased learning and memory abilities, poor neuronal morphology of the brain, significantly higher neuronal apoptosis rate in the brain, decreased level of serum Bcl-2, increased level of serum Bax, and significantly decreased protein levels of Notch1 and Hes1 in brain tissues. Compared with the model group, the rats in miR-9 inhibitor group showed obviously improved learning and memory abilities, improved neuronal morphology of the brain, an obviously lower neuronal apoptosis rate in the brain, increased level of serum Bcl-2, decreased level of serum Bax, and obviously increased protein levels of Notch1 and Hes1 in brain tissues. In conclusion, miR-9 inhibitor can promote the neurological function recovery and inhibit the neuronal apoptosis of depression model rats through activating the Notch signaling pathway, suggesting that miR-9 can be an important therapeutic target for depression.
\end{abstract}

Correspondence to: Dr Xinxue Gao, Department of Psychiatry, Jining Psychiatric Hospital, 1 Jidai Road, Jining, Shandong 272051, P.R. China

E-mail: qpdgt204@163.com

Key words: depression, Notch signaling pathway, apoptosis, neurons, miR-9

\section{Introduction}

Depression, also known as depressive disorder, is one of the most common mental diseases. It is a sustained and persistent emotional disorder syndrome, clinically characterized by high disability and suicide rates $(1,2)$. With the development of science and technology, the pathogenesis of depression has been deeply studied from biology, neurology and imaging science and a number of achievements have been recorded. However, there has been no complete theory that can reveal the occurrence and development of depression, and thus, its pathogenesis remains unclear $(3,4)$. Therefore, exploring the prevention and treatment of depression is the research focus worldwide $(5,6)$.

Micro ribonucleic acids (miRs) are a type of non-coding RNAs with 21-23 nucleotides in length, which are highly conserved in the biosynthetic pathway. Each miR can regulate one or more targets, and regulate the degradation of target genes or inhibit their translation into proteins $(7,8)$. According to the miR chip results, there is an abnormal expression of miRs in the brain cortex of patients who commit suicide due to depression (9). The miR-9 gene family is ubiquitous in different species, and the number and sequences of miR-9 vary from species to species. A previous study has shown that miR-9 is involved in numerous physiological and pathological processes in the body (10). Moreover, the research results of numerous studies have confirmed that miR-9 has important significance in regulating the cranial nerve and brain development $(11,12)$. Therefore, understanding and exploring the role of miRs in depression and their regulatory mechanism can provide an experimental basis for the prevention and treatment of depression.

Recently, it was demonstrated that neuronal apoptosis plays an important role in the pathogenesis of depression. The Notch signaling pathway has obvious persistent signals in the hippocampus of adult vertebrates and human, and it plays a key role in promoting neurogenesis and remodeling (13). The Notch signaling pathway is composed of the Notch receptor, Notch ligand and other regulatory molecules. When stimulated, Notch activates the downstream target basic helix-loop-helix transcription factor family, such as Hes1, Mash1 and Neurogenins, and further regulates the expression of transcription factors (14). However, there are few studies on whether miR-9 can improve the neuronal apoptosis in depression rats through regulating the Notch signaling pathway. 
In the present study, a rat model of depression was established using the chronic stress method, and the regulatory effect of miR-9 on the neuronal apoptosis in depression rats, as well as its mechanism of action, were explored.

\section{Materials and methods}

Reagents. miR-9 inhibitor was purchased from Guangzhou RiboBio Co., Ltd., hematoxylin and eosin (H\&E) staining kit from Beijing Solarbio Science \& Technology Co., Ltd. enzyme-linked immunosorbent assay (ELISA) kit from R\&D Systems, sodium dodecyl sulfate-polyacrylamide gel electrophoresis (SDS-PAGE) loading buffer from Shanghai Beyotime Institute of Biotechnology, terminal deoxynucleotidyl transferase-mediated dUTP nick end labeling (TUNEL) kit from Nanjing KeyGen Biotech Co., Ltd.

Instruments. Water bath kettle was purchased from Thermo Fisher Scientific, Inc., the ice machine from Grant Ice Systems (NingBo), the inverted fluorescence microscope from Nikon Corp., the water maze from Jiangsu SANS Biological Technology Co. Ltd., the western blot electrophoresis apparatus and the membrane transfer machine from Bio-Rad Laboratories, Inc.

Rats. A total of 30 specific pathogen-free male Sprague-Dawley rats, aged 12 weeks old, and weighing 220-260 g, were purchased from the Animal Center of Jining Psychiatric Hospital (Jining, China). They had good general conditions, and were adaptively fed in separate cages for 7 days under $12 / 12 \mathrm{~h}$ light/dark cycle. The rat model of depression was established using the chronic stress method, as follows: Deprivation of food for $24 \mathrm{~h}$, deprivation of water for $24 \mathrm{~h}$, swimming in ice water at $4^{\circ} \mathrm{C}$ for $5 \mathrm{~min}$, tail clamping for $5 \mathrm{~min}$, cage tilt for $45^{\circ}$ for $12 \mathrm{~h}$, and flash stimulation for $2 \mathrm{~h}$. Two to three types of stress were induced every day for 21 consecutive days. The miR-9 inhibitor was injected via caudal vein in the rats of the miR-9 inhibitor group, while normal saline was given to the rats of the control group. The study was approved by the Animal Ethics Committee of Jining Psychiatric Hospital.

Detection of learning and memory abilities via water maze test. The learning and memory abilities of rats in each group were detected via water maze test for a total of 5 days. All rats were subjected to positioning training and space exploration on the first 4 days, and those who failed to find the platform within $60 \mathrm{sec}$ were guided. Then, on day 5 , the time of crossing the original platform within $60 \mathrm{sec}$, and the residence time in the original quadrant were recorded.

Detection of neuronal morphology of brain tissues via $H \& E$ staining. Brain tissues were collected from rats and were immersed in $80 \%$ ethanol overnight, fixed with $4 \%$ paraformaldehyde, embedded in paraffin, and sliced into $8 \mu \mathrm{m}$-thick sections. According to the manufacturer's instructions of the H\&E staining kit, the sections were permeabilized with xylene, deparaffinized with ethanol at different concentrations, added dropwise with $\mathrm{H} \& \mathrm{E}$ dye and resin adhesive, and after being covered with the cover glass, observation of staining was followed.
Detection of neuronal apoptosis in the brain using TUNEL staining. Brain tissues of rats were collected and treated as aforementioned. The sections were fixed using $4 \%$ paraformaldehyde at $20^{\circ} \mathrm{C}$ for $30 \mathrm{~min}$. Next, the sections were added drop-wise with TdT fluorescein-labeled dUTP solution and were incubated in the dark. A total of $50 \mu \mathrm{l}$ TUNEL reagent (cat. no. C1088; Beyotime Institute of Biotechnology) was added to each section for incubation at $37^{\circ} \mathrm{C}$ for $60 \mathrm{~min}$. Nuclear staining reagent and mounting medium were used according to the manufacturer's instructions of the TUNEL kit. After staining, the cells were observed in five randomly-selected fields under a fluorescent microscope (IX70; Olympus Corp.). The cells were stained green if apoptosis occurred, and the apoptosis rate was calculated according to the fluorescence.

Determination of serum B-cell lymphoma-2 (Bcl-2) and Bcl-2 associated X protein (Bax) levels via ELISA. The kits were taken and equilibrated at room temperature for $30 \mathrm{~min}$. Blood samples were collected and placed at room temperature. The standards were diluted at 1:5 into standard solution, and the standard curves were plotted. The samples were added into each well repeatedly for 3 times, and $50 \mu \mathrm{l}$ of streptavidin solution were also added, followed by incubation at $37^{\circ} \mathrm{C}$ for $1 \mathrm{~h}$. Then, the plate was washed with washing liquid and patted dry. Fifty microliters of developing solution A and $50 \mu \mathrm{l}$ of developing solution $\mathrm{B}$ were added for incubation at $37^{\circ} \mathrm{C}$ for $10 \mathrm{~min}$ in the dark, and the reaction was terminated using stop buffer. Finally, the absorbance of each well was measured. Rat Bcl-2 ELISA kit (cat. no. E-EL-R0096c) and rat Bax ELISA kit (cat. no. E-EL-R0098c) were purchased from Elabscience Biotechnology Co., Ltd.

Determination of protein levels of Notchl and Hes 1 in brain tissues using western blot analysis. The brain tissues of rats were lysed using a protein lysis buffer (cat. no. QC25-05099; Shanghai Qincheng Biotechnology, Co. Ltd.) and the protein concentration was determined. BSA blocking buffer $(5 \%)$ was used as the blocking reagent. $5 \mathrm{X}$ protein loading buffer was added for water bath at $100^{\circ} \mathrm{C}$ for $5 \mathrm{~min}$. Total protein concentration was calculated by BCA protein assay kit (Pierce; Thermo Fisher Scientific, Inc.). After $10 \%$ SDS-PAGE, $30 \mu \mathrm{g}$ of protein were transferred onto a polyvinylidene difluoride membrane via a wet process for $1 \mathrm{~h}$ under $120 \mathrm{~V}$, and were sealed with $5 \%$ skim milk powder for $1 \mathrm{~h}$. The membranes were incubated with rabbit anti-Notch1 $(1: 1,000)$ and Hes1 $(1: 1,000)$ primary antibodies at $4^{\circ} \mathrm{C}$ overnight. Following the primary incubation, membranes were incubated with secondary goat anti-rabbit (HRP) IgG antibody for $1 \mathrm{~h}$. Immunoreactive bands were visualized by an enhanced chemiluminescence detection kit (Amersham; GE Healthcare). The membranes were scanned for image development, and the optical density was analyzed using ImageJ software (National Institutes of Health). Primary rabbit monoclonal anti-Notch1 antibody (dilution: 1/1,000; cat. no. ab194123), rabbit monoclonal Hes1 antibody (dilution: $1 / 1,000$; cat. no. ab221788), rabbit polyclonal $\beta$-actin antibody (dilution: 1/1,000; cat. no. ab8227), and secondary goat anti-rabbit (HRP) IgG antibody (dilution: 1/2,000; cat. no. ab6721) were all purchased from Abcam. 
A

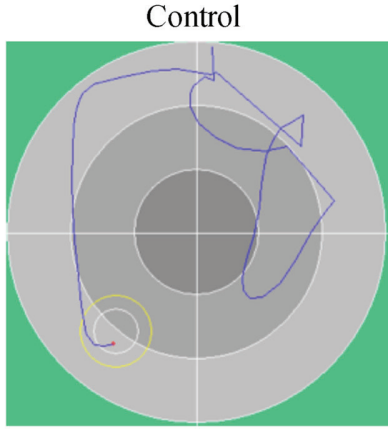

B

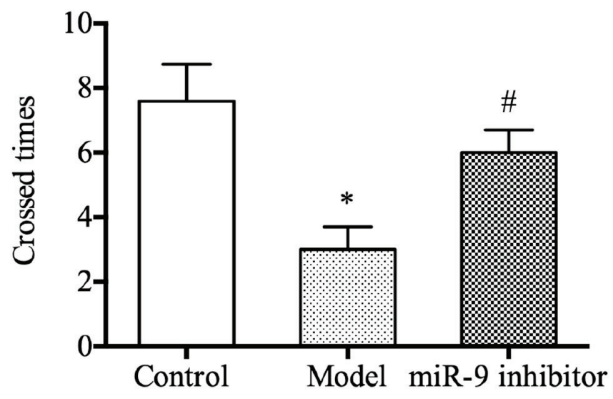

Model

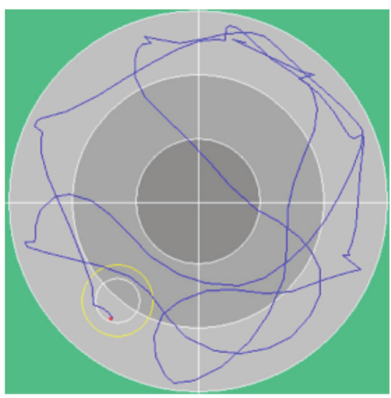

$\mathrm{C}$
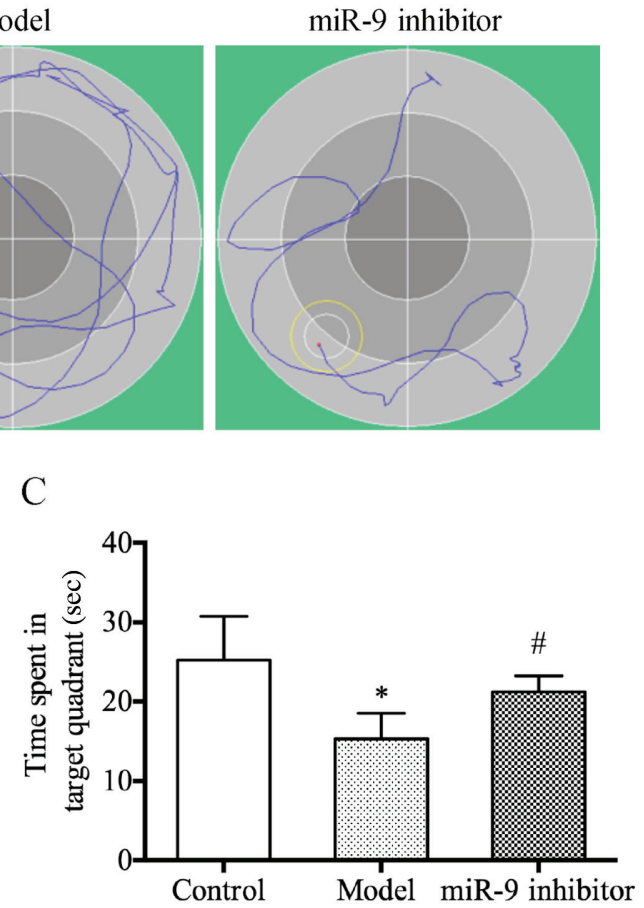

Figure 1. Learning and memory abilities of rats detected via water maze test. (A) Schematic diagram of water maze test. (B) Times of crossing the platform. (C) Residence time in the original quadrant. ${ }^{*} \mathrm{P}<0.05$, model group vs. control group; ${ }^{*} \mathrm{P}<0.05$, miR-9 inhibitor group vs. model group.

Control

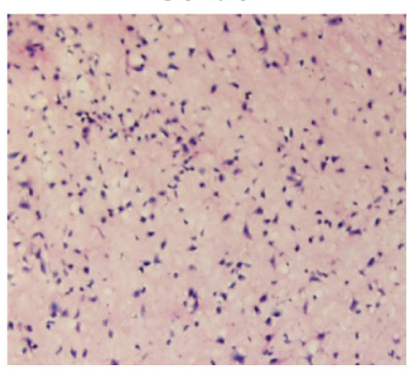

Model

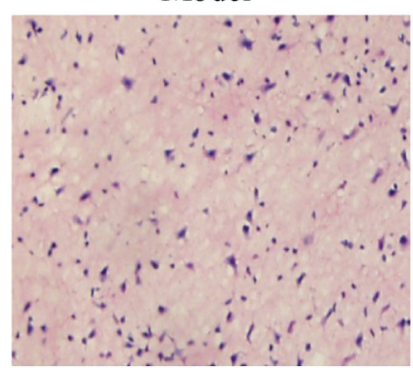

miR-9 inhibitor

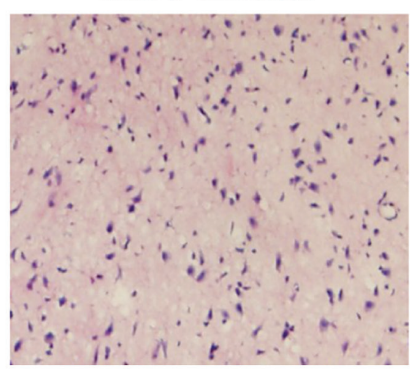

Figure 2. Neuronal morphology of the brain of rats with depression (x20).

Statistical analysis. GraphPad 5.0 software (GraphPad Software, Inc.) was used for statistical analysis. The experimental data were expressed as mean \pm standard deviation. Differences between two groups were analyzed using the Student's t-test. Comparison between multiple groups was made using one-way ANOVA followed by a post hoc test (Least Significant Difference). $\mathrm{P}<0.05$ was considered to indicate a statistically significant difference.

\section{Results}

miR-9 inhibitor could improve the learning and memory abilities of depression rats. The results of water maze test revealed that the times of crossing the original platform and the residence time in the original quadrant were significantly decreased in the model group compared with those in the control group $(\mathrm{P}<0.05)$ (Fig. 1A); whereas, they were significantly increased in miR-9 inhibitor group compared with those in the model group $(\mathrm{P}<0.05)$ (Fig. $1 \mathrm{~B}$ and $\mathrm{C})$. The above results indicate that miR-9 inhibitor can improve the learning and memory abilities of depression rats.

miR-9 inhibitor could ameliorate the neuronal morphology of the brain of depression rats. According to the results of $\mathrm{H} \& \mathrm{E}$ staining (Fig. 2), the neurons in cortical tissues shrunk, the number of neurons was reduced, and they were arranged disorderly in the model group compared with those in the control group. Compared with the model group, the neurons in cortical tissues were relaxed, the number of neurons was increased, and they were arranged orderly in the miR-9 inhibitor group. The above findings suggest that miR-9 inhibitor can ameliorate the neuronal morphology of the brain of depression rats.

miR-9 inhibitor could inhibit the neuronal apoptosis in depression rats. The results of TUNEL staining revealed that the number of apoptotis neurons was obviously larger in the model group than that in the control group $(\mathrm{P}<0.05)$ (Fig. 3A); whereas, it was obviously smaller in the miR-9 inhibitor group 

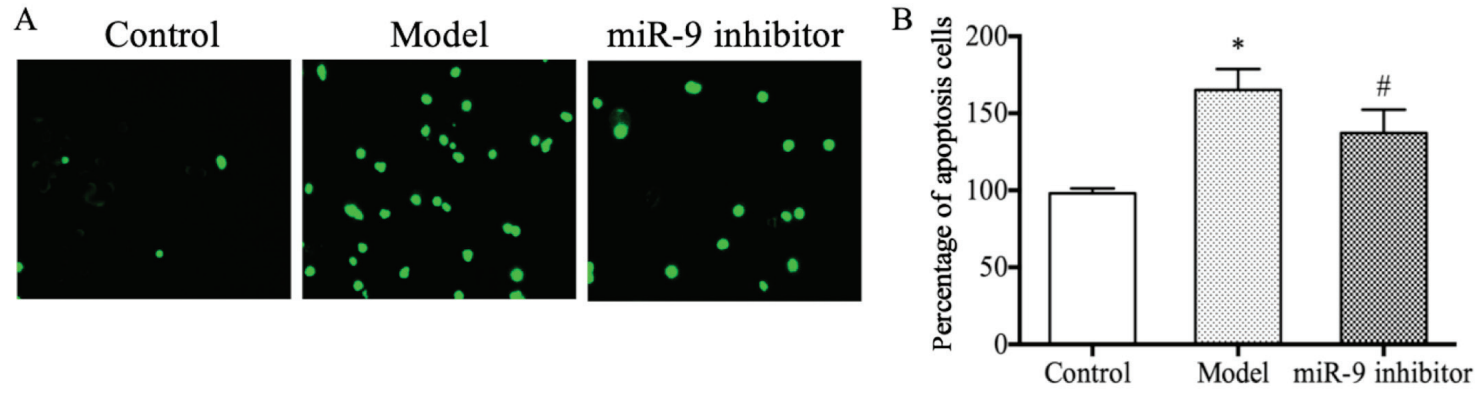

Figure 3. Neuronal apoptosis. (A) TUNEL staining (x20). (B) Apoptosis rate. " $\mathrm{P}<0.05$, model group vs. control group; $\mathrm{P}<0.05$, miR-9 inhibitor group vs. model group.
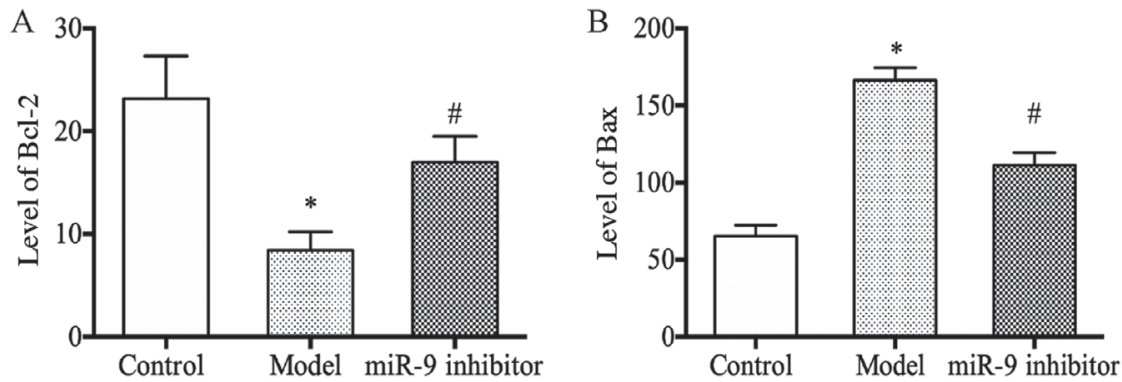

Figure 4. Levels of serum Bcl-2 and Bax in rats. (A) Bcl-2 expression level. (B) Bax expression level. ${ }^{~} \mathrm{P}<0.05$, model group vs. control group; ${ }^{*} \mathrm{P}<0.05$, miR-9 inhibitor group vs. model group. Bax, Bcl-2 associated X protein; Bcl-2, B-cell lymphoma-2.

A

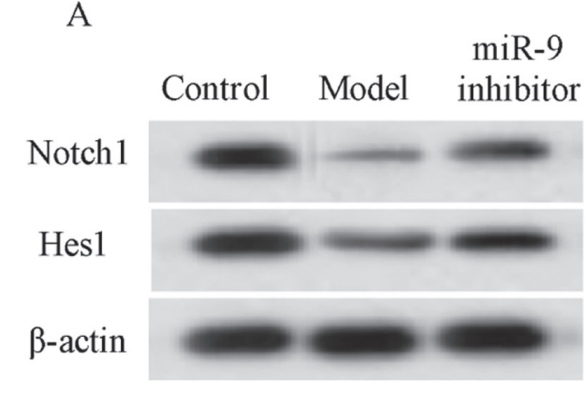

B

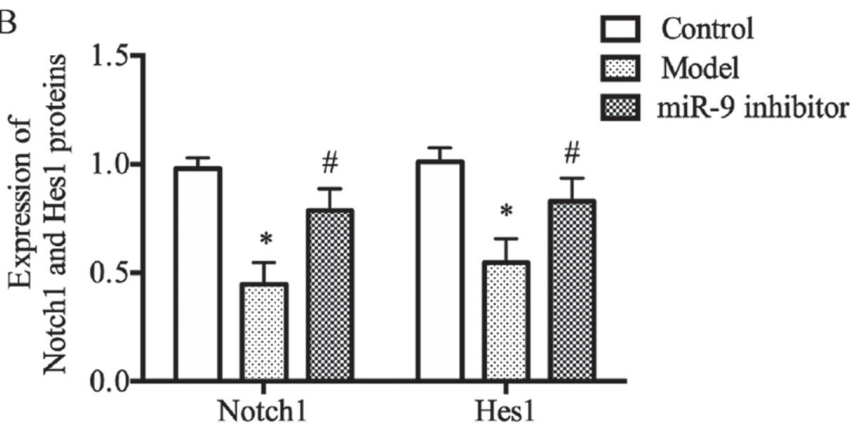

Figure 5. Protein levels of Notch1 and Hes1 in brain tissues. (A) Western blot bands. (B) Densitometry of western blots. * $\mathrm{P}<0.05$, model group vs. control group; ${ }^{\#} \mathrm{P}<0.05$, miR-9 inhibitor group vs. model group.

compared with than in the model group $(\mathrm{P}<0.05)$ (Fig. 3B), suggesting that miR-9 inhibitor can inhibit neuronal apoptosis in depression rats.

miR-9 inhibitor could upregulate the serum Bcl-2 level and downregulate the Bax level in depression rats. As shown in Fig. 4A and B, the model group presented a decreased level of serum Bcl-2 and an increased level of serum Bax compared with those in the control group $(\mathrm{P}<0.05)$; whereas, in the miR-9 inhibitor group there was observed an increased level of serum Bcl-2 and a decreased level of serum Bax compared with those in the model group $(\mathrm{P}<0.05)$.

miR-9 inhibitor could increase the protein levels of Notch1 and Hesl in brain tissues of depression rats. The results of western blot analysis revealed that model group had evidently decreased protein levels of Notch1 and Hes1 in brain tissues compared with those in the control group $(\mathrm{P}<0.05)$ (Fig. 5A); whereas, miR-9 inhibitor group had evidently increased protein levels of Notch1 and Hes1 in brain tissues compared with those in the model group $(\mathrm{P}<0.05)$ (Fig. 5B), demonstrating that the miR-9 inhibitor can increase the protein levels of Notch1 and Hes1 in brain tissues of depression rats.

\section{Discussion}

Depression is known to severely affect the human health around the world. According to the report of the World Health Organization, the depression patients account for $20 \%$ of the global population, with a dramatically increasing annual incidence rate $(15,16)$. Depression patients often live their life in a depressive mood, often have low spirits, and even feel inconsolable and profoundly pessimistic. Depression affects patients and their families in different ways, seriously 
troubling the patients' lives, learning and work, bringing a heavy burden to families and society. Therefore, finding appropriate and safe treatment means and solutions is the only way to solve the problem of depression.

In recent years, it has been shown that miRs may play an important regulatory role in the occurrence of neurological diseases, such as depression. miR-9 is a type of abnormally expressed miR. Katz et al (17) have shown that miR-9 can stimulate the proliferation of neural stem cells. Coolen et al (18) have also reported that miR-9 plays a key role in nerve regeneration and nerve repair. Moreover, Li et al (19) showed that miR-9 expression was downregulated in an in vitro cell model of Alzheimer's disease, and miR-9 was shown to significantly inhibit the differentiation of neural stem cells whose mechanism may be related to the regulation on Notch signaling pathway. The above results confirm that miR-9 is closely related to the development of cranial nerves; however, the regulatory effect and regulatory mechanism of miR-9 in depression are still unclear.

In the present study, a rat model of depression was established using the chronic stress method, and the miR-9 inhibitor was used for intervention. First, the water maze test, an experimental method to detect the learning and memory abilities of rats or mice (20), was performed. It was found that the escape latency and residence time significantly declined in the model group compared with those in the control group; whereas, they were significantly increased in the miR-9 inhibitor group compared with those in the model group, indicating that miR-9 inhibitor can improve the learning and memory abilities of depression rats. Then, the changes in the neuronal morphology of the brain and the neuronal apoptosis were determined. The results revealed that in miR-9 inhibitor group, the neuronal morphology in the brain was obviously improved, the number of neurons was increased and they were arranged orderly, and the number of apoptosis neurons was obviously reduced, suggesting that the miR-9 inhibitor can remarkably improve the cranial nerve function and suppress the neuronal apoptosis in depression rats. In addition, the levels of serum Bcl-2 and Bax were detected. It was confirmed that miR-9 inhibitor group had an increased level of serum Bcl-2 and a decreased level of serum Bax, proving that miR-9 inhibitor can inhibit apoptosis. To further explore the mechanism of action of miR-9 inhibitor, the protein levels of Notch1 and Hes1 in the brain were determined using western blot analysis. The results revealed that miR-9 inhibitor group had evidently increased protein levels of Notch1 and Hes1 in the brain compared with those in the model group, indicating that miR-9 inhibitor can exert a therapeutic effect on depression rats through activating the Notch signaling pathway.

In conclusion, the results of this study demonstrate that miR-9 inhibitor can improve the neuronal morphology, ameliorate the neurological function and inhibit the apoptosis in depression rats, and the mechanism may be related to the activation of the Notch signaling pathway. The present study provides a new perspective for the treatment of depression and an experimental basis for the application of miR-9 inhibitor in the prevention and diagnosis of depression.

\section{Acknowledgements}

Not applicable.

\section{Funding}

No funding was received.

\section{Availability of data and materials}

All data generated or analyzed during this study are included in this published article.

\section{Authors' contributions}

PX, XG and YL designed the study and performed the experiments. PX and XZ established the animal models. ZM and SS analyzed the data. PX and XG prepared the manuscript. All authors read and approved the final manuscript.

\section{Ethics approval and consent to participate}

The study was approved by the Animal Ethics Committee of Jining Psychiatric Hospital (Jining, China).

\section{Patient consent for publication}

Not applicable.

\section{Competing interests}

The authors declare that they have no competing interests.

\section{References}

1. Smith K: Mental health: A world of depression. Nature 515: 181, 2014.

2. Liu J, Xu ZQ, Yi X, Wang YJ and Zhou HD: A study on related factors of hemodynamic depression in carotid artery stenting. Eur Rev Med Pharmacol Sci 22: 5255-5263, 2018.

3. Anthes E: Depression: A change of mind. Nature 515: 185-187, 2014.

4. Rahim T and Rashid R: Comparison of depression symptoms between primary depression and secondary-to-schizophrenia depression. Int J Psychiatry Clin Pract 21: 314-317, 2017.

5. Monteggia LM, Malenka RC and Deisseroth K: Depression: The best way forward. Nature 515: 200-201, 2014.

6. Hammen C: Risk factors for depression: An autobiographical review. Annu Rev Clin Psychol 14: 1-28, 2018.

7. Hu Z, Jiang Y, Huo X, Yang Y, Davies H, Botchway BO and Fang M: Prospective role of MicroRNAs in depression. Curr Med Chem 24: 3508-3521, 2017.

8. Tavakolizadeh J, Roshanaei K, Salmaninejad A, Yari R, Nahand JS, Sarkarizi HK, Mousavi SM, Salarinia R, Rahmati M, Mousavi SF, et al: MicroRNAs and exosomes in depression: Potential diagnostic biomarkers. J Cell Biochem 119: 3783-3797, 2018.

9. Lopez JP, Kos A and Turecki G: Major depression and its treatment: microRNAs as peripheral biomarkers of diagnosis and treatment response. Curr Opin Psychiatry 31: 7-16, 2018.

10. Ma ZY, Chen F, Xiao P, Zhang XM and Gao XX: Silence of miR-9 protects depression mice through Notch signaling pathway. Eur Rev Med Pharmacol Sci 23: 4961-4970, 2019.

11. Sim SE, Lim CS, Kim JI, Seo D, Chun H, Yu NK, Lee J, Kang SJ, Ko HG, Choi JH, et al: The brain-enriched MicroRNA miR-9-3p regulates synaptic plasticity and memory. J Neurosci 36: 8641-8652, 2016.

12. Madelaine R, Sloan SA, Huber N, Notwell JH, Leung LC, Skariah G, Halluin C, Paşca SP, Bejerano G, Krasnow MA, et al: MicroRNA-9 couples brain neurogenesis and angiogenesis. Cell Rep 20: 1533-1542, 2017.

13. Ables JL, Breunig JJ, Eisch AJ and Rakic P: Not(ch) just development: Notch signalling in the adult brain. Nat Rev Neurosci 12: 269-283, 2011. 
14. Du M, Tan Y, Liu G, Liu L, Cao F, Liu J, Jiang P and Xu Y: Effects of the Notch signalling pathway on hyperoxia-induced immature brain damage in newborn mice. Neurosci Lett 653 : 220-227, 2017.

15. Cepeda MS, Katz EG and Blacketer C: Microbiome-Gut-Brain Axis: Probiotics and their association with depression. J Neuropsychiatry Clin Neurosci 29: 39-44, 2017.

16. Dusi N, Barlati S, Vita A and Brambilla P: Brain structural effects of antidepressant treatment in major depression. Curr Neuropharmacol 13: 458-465, 2015.

17. Katz S, Cussigh D, Urbán N, Blomfield I, Guillemot F, Bally-Cuif L and Coolen M: A nuclear role for miR-9 and argonaute proteins in balancing quiescent and activated neural stem cell states. Cell Rep 17: 1383-1398, 2016.
18. Coolen M, Katz S and Bally-Cuif L: miR-9: A versatile regulator of neurogenesis. Front Cell Neurosci 7: 220, 2013.

19. Li F, Chen A and Zhang J: miR-9 stimulation enhances the differentiation of neural stem cells with zoanthamine by regulating Notch signaling. Am J Transl Res 11: 1780-1788, 2019

20. Guo J, Chang L, Li C, Li M, Yan P, Guo Z, Wang C, Zha Q and Wang Q: SB203580 reverses memory deficits and depression-like behavior induced by microinjection of A $\beta 1-42$ into hippocampus of mice. Metab Brain Dis 32: 57-68, 2017.

(c) (i) () () This work is licensed under a Creative Commons EY No ND Attribution-NonCommercial-NoDerivatives 4.0 International (CC BY-NC-ND 4.0) License. 